\title{
Private Edge Domination Number of a Graph
}

\author{
Robinson C. $S^{1}$ and Kavitha $S^{2 *}$ \\ ${ }^{1}$ Department of Mathematics, Scott Christian College, Nager Coil-629001, India \\ ${ }^{2}$ Department of Mathematics, Arunachala College of Engineering for Women, Nagercoil, India
}

Received 3 October 2012, accepted in final revised form 8 April 2013

\begin{abstract}
A set $S \subseteq E$ is said to be a private edge dominating set, if it is an edge dominating set, for every $e \in S$ has at least one external private neighbor in $E \backslash S$. Let $\gamma_{p v t}^{\prime}(G)$ and $\Gamma_{p v t}^{\prime}(G)$ denote the minimum and maximum cardinalities, respectively, of a private edge dominating sets in a graph $G$. In this paper we characterize connected graph for which $\Gamma_{p v t}^{\prime}(G) \leq q / 2$ and the graph for some upper bounds. The private edge domination numbers of several classes of graphs are determined.

Keywords: Edge domination; Perfect domination; Private domination; Edge irredundant sets.

(C) 2013 JSR Publications. ISSN: 2070-0237 (Print); 2070-0245 (Online). All rights reserved. doi: http://dx.doi.org/10.3329/jsr.v5i2.12024 J. Sci. Res. 5 (2), 283-294 (2013)
\end{abstract}

\section{Introduction}

Let $G=(V, E)$ be a simple connected graph [1] with $|\mathrm{V}|=p$ and $|E|=q, q \geq 2$. A set $S \subseteq E$ is an edge dominating set if each edge in $E$ is either in $S$ or is adjacent to an edge in $S$ and is an independent edge dominating set if edges of $S$ are independent. The edge domination number $\gamma^{\prime}(G)$ is the minimum cardinality among all minimal edge dominating sets, and $\Gamma^{\prime}(G)$ is the maximum cardinality among all minimal edge dominating sets of $\mathrm{G}$ [2]. The minimum and the maximum cardinalities taken over all maximal independent edge dominating sets of $G$ is denoted by $\mathrm{i}^{\prime}(G)$ and $\beta(G)$. The open neighborhood of an edge $e$, denoted $N(e)$, is the set $\left\{e^{\prime} \in E: e\right.$ is adjacent to $\left.e\right\}$ and the closed neighborhood of $e$, denoted $N[e]=\{e\} \cup N(e)$ For $e \in S \subseteq E$, we define $P_{n}[e, S]=N[e]-N[S-\{e\}]$. If $P_{n}[e, S] \neq \Phi$ then $e$ is said to be an edge irredundant in $S$. The set $S \subseteq E$ is said to be an edge irredundant set if $P_{n}[e, S] \neq \Phi$ for all $e \in S$. The

*Corresponding author: kavithasadasivan@gmail.com 
minimum and maximum cardinalities taken over all maximal edge irredundant sets of edges of $G$ is $\operatorname{ir}^{\prime}(G)$ and $\operatorname{IR}^{\prime}(G)$, respectively [2]. If $P_{n}(e, S)=N(e)-N(S-\{e\})=e^{\prime}$, then the edge $e^{\prime}$ is an external edge private neighbor of $e$.

An edge subset $S$ in a graph $G$ is said to be perfect edge dominating set in $G$ if each edge of the complementary graph $E \backslash S$ of $S$ in $G$ is adjacent to exactly one edge in $S$. The minimum cardinality among the perfect edge dominating sets in a graph $G$ is denoted by $\gamma_{\mathrm{p}}(\mathrm{G})$. A set $S \subseteq E$ is said to be a private edge dominating set if it is an edge dominating set, every $e \in S$ has at least one external private neighbor in $E \backslash S$.The minimum and maximum cardinalities taken over all private edge dominating sets in a graph $G$ is called a private edge domination numbers $\gamma_{p v t}^{\prime}(G)$ and $\Gamma_{p v t}^{\prime}(G)$. It can be observed that the minimum cardinality of a private dominating set is always equal to $\gamma^{\prime}(G)$. For a real number $x,\lfloor x\rfloor$ denotes the largest integer not greater than $x$ and $\lceil x\rceil$ denotes the smallest integer not less than $x$. An edge dominating set $S$ is a minimal edge dominating set if no proper subset $S^{\prime} \subset S$ is an edge dominating set. An edge dominating set $S$ is a maximal edge dominating set if no super subset $S^{\prime} \supset S$ is an edge dominating set. The degree of an edge $e=u v$ is defined to be $\operatorname{deg}(u)+\operatorname{deg}(v)-2$.

The notion of private dominating set has been introduced as a concept by Bollobas and Cockayane [3] . Further studied by B.J Prasad and etl [5]. In this paper we carried out private domination number for edge set of a graph. Also we characterize certain properties of private edge domination number, and we obtain certain bounds and connection with other edge domination related parameters.

\section{Some Basic Results}

\section{Theorem (Existence Theorem)}

2.1: A graph $G$, without isolated edges and $q \geq 2$ has a minimum edge dominating set which is also a private edge dominating set.

Proof. Let $S$ be a $\gamma^{\prime}(G)$ set for which the number of edges in $\langle S\rangle$ having an open private edge neighbor is maximum. If an edge $e^{\prime} \in S$ does not have an open private edge neighbor, then it must be isolated in $\left\langle S>\right.$ Since $G$ has no isolated edge, $\mathrm{e}^{\prime}$ must be adjacent at least one edge say $e^{\prime \prime}$ in $E \backslash S$. But in this case $S \backslash\left\{\mathrm{e}^{\prime}\right\} \cup\left\{\mathrm{e}^{\prime \prime}\right\}$ is a minimum edge dominating set, in which an edge $\mathrm{e}^{\prime \prime}$ has an edge $\mathrm{e}^{\prime}$ as an open private edge neighbor, contradiction to the minimality of a number of edges in $S$ having an open private edge neighbor. Thus $S$ must be a Private edge dominating set.

\section{Observations 2.2:}

1. For any graph $G, \gamma^{\prime}(G) \leq \Gamma_{p v t}^{\prime}(G)$.

2. For any complete graph $K_{p}$ with $p \geq 3 \quad \Gamma_{p v t}^{\prime}(G)=\left\lfloor\frac{P}{2}\right\rfloor$.

3. For any path $P_{p}, \Gamma_{p v t}^{\prime}(G)=\left\lfloor\frac{q}{2}\right\rfloor$ 
4. For any bipartite graph complete graph $K_{m, n} m, n \geq 2, \Gamma_{p v t}^{\prime}(G)=\max \{m, n\}$.

5. For any graph $G, 1 \leq \Gamma_{p v t}^{\prime}(G) \leq\left\lfloor\frac{q}{2}\right\rfloor$.

6. For any graph $G, \Gamma_{p v t}^{\prime}(G) \leq \Gamma^{\prime}(G) \leq I R^{\prime}(G)$.

Theorem 2.3: For any connected graph $\mathrm{G},|\mathrm{S}|=\gamma^{\prime}(\mathrm{G})$, then $\Gamma_{p v t}^{\prime}(G) \leq \sum_{e \in S} d(e)$.

Proof. Let $S$ be a $\Gamma_{p v t}^{\prime}(G)$ set of a graph $G$. Suppose $e_{1} \in S$ there exist an edge $e_{2} \in E \backslash S$, satisfying $N\left(e_{2}\right) \cap S=\left\{e_{1}\right\}$. Then $E \backslash S$ is a dominating set of $S$.

$$
\gamma^{\prime}(G) \leq|E \mathrm{Q}| \leq \mathrm{q}-\Gamma_{p v t}^{\prime}(G)
$$

Also we have in ref. [4] $|E(G)| \mid \gamma^{\prime}(\mathrm{G}) \leq \sum_{e \in S} d(e), \gamma^{\prime}(\mathrm{G}) \geq q-\sum_{e \in S} d(e)$

Now from (1) $q-\sum_{e \in S} d(e) \leq q-\Gamma_{p v t}^{\prime}(G)$. Therefore $\Gamma_{p v t}^{\prime}(G) \leq \sum_{e \in S} d(e)$

Theorem 2.4: Every minimal private edge dominating set is edge dominating and edge irredundant.

Proof. Let $G$ be a graph, $S$ be a minimal private edge dominating set of $G$, which implies that every element of $S$ contains at least one external private edge neighbor. This implies $S$ is edge irredundant.

Remark 2.5. Converse of the Theorem 2.4 is not true.

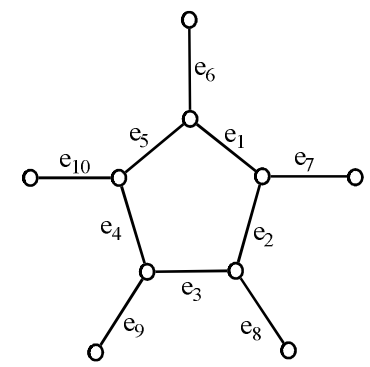

Fig. 1.

In Fig. $1 S=\left\{e_{6}, e_{7}, e_{8}, e_{9}, e_{10}\right\}$ is edge irredundant and edge dominating, but not private edge dominating set.

Theorem 2.6: Every minimal private edge dominating set is maximal irredundant edge set of $G$.

Proof. Assume that $S$ is a minimal private edge dominating set. To show that $S$ is a maximal edge irredundant set of $G$. Suppose it is not true, that is if $S$ is not a maximal 
irredundant edge set, there must exist an edge $e_{1} \in E \backslash S$ for which $S \cup\left\{e_{1}\right\}$ is irredundant. This means in particular that $P_{n}\left[e_{1}, S \cup\left\{e_{1}\right\}\right] \neq \Phi$. i.e there exists at least one edge $e_{2}$ which is a private edge neighbor of $e_{1}$ with respect to $S \cup\left\{e_{1}\right\}$. But this means that no edge in $S$ is adjacent to $e_{2}$, that is $S$ is not a dominating set. This contradicts that $S$ is edge dominating set.

Remark 2.7: But the converse of the Theorem 2.6 is not true.

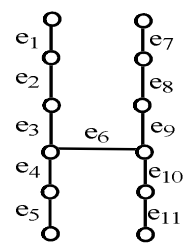

Fig.2

In Fig. $2 S=\left\{e_{2}, e_{3}, e_{8}, e_{9}\right\}$ is a maximal edge irredundant set not minimal private edge dominating set.

Theorem 2.8: For every $\Gamma^{\prime}{ }_{p v t}(G)$-set is a minimal edge dominating set.

Proof. . Let $S$ be $\Gamma^{\prime}{ }_{p v t}(G)$ a set of a graph $G$. Then every edge in $S$ has an external private edge neighbor in $E \backslash S$. Hence $S$ is a minimal edge dominating set of $G$.

Remark 2.9: The converse of the Theorem 2.8 is not true.

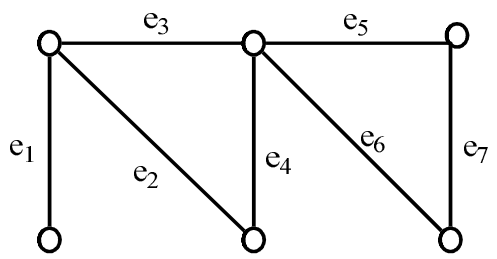

Fig. 3

In Fig.3 $S=\left\{e_{1}, e_{4}, e_{7}\right\}$ is a minimal edge dominating set but not a private edge dominating set.

\section{Bounds for Private Edge Domination Number}

Theorem 3.1: For any tree $T, \Gamma_{p v t}^{\prime}(G) \leq \mathrm{q}-\Delta^{\prime}$ Equality holds for wounded spider and star graphs. 
Proof. Let $e^{\prime}$ be any edge having maximum degree $\Delta^{\prime}$ in a tree $T$. Let $S$ be maximum private edge dominating set of $G$. Suppose $e^{\prime} \in S$. If all the edges adjacent to $e^{\prime}$ are in $E \backslash S$ then we are through. Otherwise if some of its neighbor is in $S$ the corresponding to each one of them there will be at least one edge in $E \backslash S$ and hence the theorem follows. Hence in these case $|E \backslash S| \geq \Delta^{\prime}$ Further suppose $\mathrm{e}^{\prime}$ not in $S$, in this case for each $e^{\prime \prime} \in N\left(e^{\prime}\right) \cup S$ then there exits an external private neighbor in $E \backslash S$. Hence $|E \backslash S| \geq \Delta^{\prime}$. It is easy to see that the equality holds for wounded spider and star graphs.

Theorem 3.2: Let $S$ be a $\Gamma_{p v t}^{\prime}(G)$ set of a graph $G$, for any edge $e \in S$,

$$
\Gamma_{p v t}^{\prime}(G) \leq \mathrm{q}-\operatorname{deg}(\mathrm{e})
$$

Proof. Let $S$ be $\Gamma_{p v t}^{\prime}(G)$-set of $G$. Let the degree of $e$ be $k_{1}$. Assume that $e$ is adjacent to $k$ edges in $\mathrm{S}$, then the edge $e$ is adjacent to $k_{1}-k$ edges in $E \backslash S$.

If $k>0$, then each neighbor of $e$ in $S$ must have an external private neighbor in $E \backslash S$, and these edges must be distinct and so $|E \backslash S| \geq\left(k_{1}-k\right)+k=d(e)$. Therefore $\Gamma_{p v t}^{\prime}(G) \leq q-\operatorname{deg}(e)$

$$
\text { If } k=0 \text {, then }|E \backslash S| \geq k_{1}=d(e) \text {. Hence } \Gamma_{p v t}^{\prime}(G) \leq q-\operatorname{deg}(e)
$$

Remark 3.3: It is easy to see that equality holds for wounded spider and star graphs.

Theorem 3.4: For a graph $G$, an edge dominating set $S$ and its complement $E \backslash S$ are private edge dominating set in $G$ if and only if $\Gamma_{p v t}^{\prime}(G)=\frac{q}{2}$.

Proof. Clearly $|E|$ is even $S$ is a $\Gamma_{p v t}^{\prime}(G)$ set such that $|S|=\frac{q}{2}$. This implies that $|E \backslash S|=\frac{q}{2}$, for every $e \in S$ there exists a unique edge $e^{\prime} \in E \backslash S$ adjacent to $e$ and hence $|E \backslash S|$ also a private edge dominating set of $G$. Conversely suppose $S$ and $E \backslash S$ are private edge dominating set of $G$, then $|S| \leq \frac{q}{2}$ and $|E \backslash S| \leq \frac{q}{2}$. If $|S|<\frac{q}{2}$ then $|E \backslash S|>\frac{q}{2}$, which implies that $E \backslash S$ is not a private edge dominating set, a contradiction. Hence $|S|=\frac{q}{2}$.

Remark 3.5: If $G$ has odd number of edges, then both edge dominating sets and its complement cannot be private edge dominating set of $G$.

Theorem 3.6: Let $G$ be a graph, suppose a minimal edge dominating set $S$ of a graph $G$ is a perfect edge dominating set, then it is also a private edge dominating set of $G$.

Proof. Let $S$ be a minimal edge dominating set of $G$, which is also a perfect edge dominating set of $G$. If an edge $e \in S$ is adjacent to an edge $e_{1} \in E \backslash S$, then it will be an external private neighbor of $e$ otherwise $e$ will be adjacent to at least one edge other 
than $e_{1} \in E \backslash S$, which is a contradiction to the definition of perfect edge dominating set. Also if $e \in S$ is adjacent to no edge in $E \backslash S$, then $E \backslash\{e\}$ is an edge dominating set of $G$, which is a contradiction to the minimality.

Remark 3.7: Converse of the Theorem 3.6 is not true.

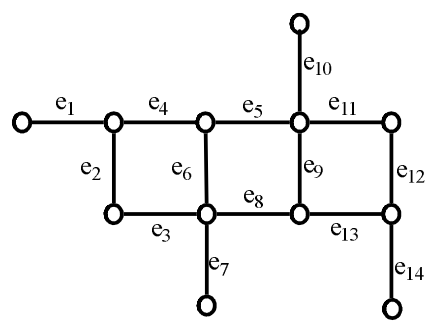

Fig. 4

In Fig. $4, S=\left\{e_{1}, e_{3}, e_{11}, e_{14}\right\}$ is a minimal edge dominating set and private edge dominating set but not perfect.

Theorem 3.8: Let $S$ be a perfect edge dominating set of a graph $G, S$ is a private edge dominating set of $G$ if and only if $E \backslash S$ is edge dominating set of $G$.

Proof. Let $S$ be a perfect edge dominating set of a graph $G$. Assume that $S$ is a private edge dominating set of $G, S$ is a minimal edge dominating set of $G$.Therefore $E \backslash S$ is edge dominating set of $G$.Conversely suppose that $E \backslash S$ is an edge dominating set of $G$, since $S$ is a perfect edge dominating set of $G$, so every edge in $E \backslash S$ is adjacent to unique element in $S$, and every edge in $S$ is adjacent to at least one edge in $E \backslash S$.Hence every edge in $E \backslash S$ is a private edge neighbor of a edge in $S$. So $S$ is a private edge dominating set of $G$.

Remark 3.9: If we delete perfect, Theorem 3.8 does not need to be true.

Theorem 3.10: For any connected graph $G, \Gamma_{p v t}^{\prime}(G) \leq \Gamma^{\prime}(G)$, where $\Gamma^{\prime}(G)$ is the upper domination number.[2].

Theorem 3.11: For any connected graph $G, \Gamma_{p v t}^{\prime}(G)+\gamma^{\prime}(G) \leq q$.

Proof. Let $S$ be a minimal private edge dominating set with maximum cardinality. Then $E \backslash S$ is an edge dominating set. Hence $|\mathrm{E} \backslash \mathrm{S}| \geq \gamma^{\prime}$, then $q-|\mathrm{S}| \geq \gamma^{\prime}$

Theorem 3.12: For any connected graph $G, \Gamma_{p v t}^{\prime}(G)=\frac{q}{2}$, then $\Gamma_{p v t}^{\prime}(G)=\Gamma^{\prime}(G)$

Proof. Assume that $\Gamma_{p v t}^{\prime}(G)=\frac{q}{2}$ also $\Gamma_{p v t}^{\prime}(G) \leq \frac{q}{2}$. But by the observation $\Gamma_{p v t}^{\prime}(G) \leq \Gamma^{\prime}(G), \frac{q}{2} \leq \Gamma^{\prime}(G)$. This implies that $\frac{q}{2} \leq \Gamma^{\prime}(G) \leq \frac{q}{2}$. Therefore $\Gamma_{p v t}^{\prime}(G)$ $=\frac{q}{2}=\Gamma^{\prime}(G)$ 
Remark 3.13: The converse of the Theorem 3.12 needs not be true.

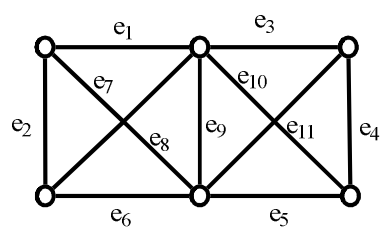

Fig. 5

In Fig. $5 S=\left\{e_{1}, e_{3}, e_{9}\right\}, \Gamma_{p v t}^{\prime}(G)=\Gamma^{\prime}(G)$ which is not equal to $\frac{q}{2}$.

Theorem 3.14: For every connected graph $G$, if the edge perfect domination number is $\frac{q}{2}$ then $\gamma_{\mathrm{P}}^{\prime}(\mathrm{G})=\Gamma_{p v t}^{\prime}(G)=\Gamma^{\prime}(G)$.

Proof. Let $G$ be a connected graph, let $S$ be a perfect edge dominating set, such that $|S|=\frac{q}{2} \quad$ By the definition of perfect dominating set $|N(e) \cap S|=1$, for all $e \in E \backslash S$ which implies that $S$ is an private edge dominating set with $|S|=\frac{q}{2}$. Always $\frac{q}{2} \leq|\mathrm{S}|$ $\leq \Gamma_{p v t}^{\prime}(G) \leq \Gamma^{\prime}(G) \leq \frac{q}{2}, \gamma_{\mathrm{P}}^{\prime}(\mathrm{G})=\Gamma_{p v t}^{\prime}(G)=\Gamma^{\prime}(G)$.

Remark 3.15: The converse of the Theorem 3.14 is not true.

In a triangle graph $\gamma_{\mathrm{P}}^{\prime}(\mathrm{G})=\Gamma_{p v t}^{\prime}(G)=\Gamma^{\prime}(G)=1$, but $\gamma_{\mathrm{P}}^{\prime}(\mathrm{G})$ is not equal to $\frac{q}{2}$.

Theorem 3.16: For any connected graph $G, S$ be a $\Gamma_{p v t}^{\prime}(G)$ set, $\gamma^{\prime}(\mathrm{G})+\Gamma_{p v t}^{\prime}(G)=q$ if and only if $E \backslash S$ is a minimum edge dominating set .

Proof. The result is obviously true.

Theorem 3.17: For any connected graph $G$, let $\mathrm{S}$ be a $\Gamma_{p v t}^{\prime}(G)$ set. If $E \backslash S$ is a minimum edge dominating set, then $\Gamma_{p v t}^{\prime}(G)=\Gamma^{\prime}(G)=\operatorname{IR}^{\prime}(\mathrm{G})=\frac{q}{2}$

Proof. Let $|\mathrm{S}|=\Gamma^{\prime}{ }_{p v t}(G)$, also $\mathrm{q}-\Gamma^{\prime}{ }_{p v t}(G)=\gamma^{\prime}(\mathrm{G})$ then $q=\gamma^{\prime}(G)+\Gamma_{p v t}^{\prime}(G)$. We show that $\Gamma_{p v t}^{\prime}(G)=\frac{q}{2}$. Suppose $\Gamma_{p v t}^{\prime}(G)<\frac{q}{2}$ which implies that $\quad \gamma^{\prime}(\mathrm{G})$ $>\frac{q}{2}$ which is a contradiction. Hence $\Gamma_{p v t}^{\prime}(G)=\Gamma^{\prime}(G)=\operatorname{IR}^{\prime}(G)=\frac{q}{2}$

Remark 3.18: The converse of the Theorem 3.17 is not true. 


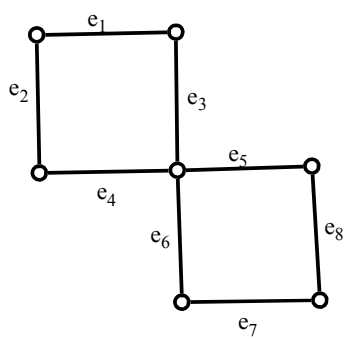

Fig. 6

In Fig. $6 S=\left\{e_{3}, e_{4}, e_{5}, e_{6}\right\}$ satisfying $\Gamma_{p v t}^{\prime}(G)=\Gamma^{\prime}(G)=\operatorname{IR}^{\prime}(G)=\frac{q}{2}$ but $E \backslash S$ is not a minimum Edge dominating set.

Theorem 3.19: For any connected graph $G, S$ be a $\gamma_{\mathrm{P}}^{\prime}(\mathrm{G})$ set, then $E \backslash S$ is a minimum edge dominating set if and only if $\gamma_{\mathrm{P}}^{\prime}(\mathrm{G})=\Gamma^{\prime}{ }_{p v t}(G)=\Gamma^{\prime}(G)=\mathrm{IR}^{\prime}(\mathrm{G})=\frac{q}{2}$.

Proof. Let $G$ be any connected graph, $S$ be a $\gamma_{\mathrm{P}}^{\prime}(G)$-set which implies that $|N(e) \cap S|=1$, for all $e \in E \backslash S$. Assume that $E \backslash S$ is a minimum edge dominating set, since $S$ be a $\gamma_{\mathrm{P}}(G)$ set which is dominating and $|N(e) \cap S|=1$, for all $e \in E \backslash S$ which implies $S$ is a minimum edge dominating set, also $P_{n}\left(e^{\prime}, S\right)=1$ for all $\mathrm{e}^{\prime} \in \mathrm{S}$. Therefore every edge in $E \backslash S$ is adjacent to exactly one edge in $S$ and every edge in $S$ is adjacent to at least one edge in $E \backslash S$.So that $|N(e) \cap S|=1$, for all $e \in E \backslash S$. Hence $\gamma_{\mathrm{P}}^{\prime}(\mathrm{G})=\frac{q}{2}=$ $\Gamma^{\prime}{ }_{p v t}(G)$. Conversely assume that $\gamma_{\mathrm{P}}^{\prime}(\mathrm{G})=\Gamma^{\prime}{ }_{p v t}(G)=\Gamma^{\prime}(G)=\operatorname{IR}^{\prime}(G)=\frac{q}{2} \quad$ so that every edge in $S$ is adjacent with exactly one edge in $E \backslash S$. This implies $E \backslash S$ is a minimum edge dominating set.

Theorem 3.20: For any $C_{4 n}, \beta(G)=\Gamma_{p v t}^{\prime}(G)=\Gamma^{\prime}(G)=\operatorname{IR}^{\prime}(\mathrm{G})=\frac{q}{2}$

Theorem 3.21: For any connected Tree, $\Delta \leq 3$ and if,$\beta(G)=\Gamma_{p v t}^{\prime}(G)$, then $\gamma_{\mathrm{P}}^{\prime}(\mathrm{G})$ $\leq \Gamma_{p v t}^{\prime}(G)$

Proof. Let $S$ be a $\Gamma_{p v t}^{\prime}(G)$ set. (Choose $S$ in such a way that $\langle S\rangle$ has minimum number of edges). Let $F$ be the set which contains external private neighbor of $S$.Let $X$ denote the edges which are neither in $S$ nor in $F$, but in $E \backslash S$.

$$
\text { So that }|E|=|S|+|F|+|X|
$$


Case (i) $|X|=\Phi$.

Therefore $|E|=|S|+|F|$ which implies every edge in $S$ is adjacent to at least one external private neighbor, $|N(e) \cap S|=1$, for all $e \in E \backslash S$

Suppose $|N(e) \cap S| \neq 1$, then $e$ is not a private neighbor of any element in $S$. This implies $e \in X,|X| \neq \Phi,|N(e) \cap S|=1$, for all $e \in E \backslash S$. So that $S$ is also a perfect edge dominating set, $\gamma_{\mathrm{P}}(G) \leq|S|=\Gamma_{p v t}^{\prime}(G)$.

Case (ii) $|X| \neq \Phi$

Let $e^{\prime} \in E \backslash S$ which implies $e^{\prime} \in E \backslash$. Therefore $\left|N\left(e^{\prime}\right) \cap S\right| \neq 1$, implies $\left|N\left(e^{\prime}\right) \cap S\right| \leq 2$, since $e^{\prime}$ is adjacent to at least two elements in $S$, also $\Delta \leq 3$ implies $\left|N\left(e^{\prime}\right) \cap S\right|=2$. Let $e_{1}, e_{2} \in S$ which are adjacent with $\mathrm{e}^{\prime}$. By the definition of $S, e_{1}$ has one external private neighbor and $e_{2}$ have exactly one external private neighbor, so that $\mathrm{e}^{\prime}$ is adjacent with four edges, two of them belongs to $S$ and two of them belongs to $E \backslash S$. Also $e_{1}$ and $e_{2}$ have at least one external private neighbor. So that $e_{1}$ and $e_{2}$ are not adjacent with any element in $S$, also $\Delta \leq 3$. Suppose if they are belongs to $S$, then $e_{1}$ and $e_{2}$ does not have any external private neighbor, also $\beta(G)>\Gamma^{\prime}{ }_{p v t}(G)$ which gives a contradiction. In $S$, we eliminate $e_{1}$ and $e_{2}$ and add $\mathrm{e}^{\prime}$, we get a perfect graph which is $\left|\mathrm{S}^{\prime}\right|<|\mathrm{S}|$. Also all elements in $\mathrm{S}^{\prime}$ with the condition that $\left|\mathrm{N}(\mathrm{e}) \cap \mathrm{S}^{\prime}\right|=1$ for all e $\in \mathrm{E} \backslash \mathrm{S}^{\prime}$. Hence $\gamma_{\mathrm{P}}^{\prime}(\mathrm{G}) \leq \Gamma^{\prime}{ }_{p v t}(G)$.

Theorem 3.22: $\quad$ In a $(p, q)$ connected graph $p \geq 3, \Gamma_{p v t}^{\prime}(G) \leq \mathrm{p}-2$.

Proof. Let $|S|=k$ be a minimal edge private dominating set with maximum cardinality. Suppose $k>p-2$ notice that no edge in $S$ can have both of its end points adjacent to a line is $S$, for such edge cannot have proper neighbor. Hence the edges in $S$ form a sub graph of $G$, which is a union of star graphs.

Suppose $S$ contains $k^{\prime}$ edges which are independent in $S$. Since each edge which is not independent is $S$ has at least one proper neighbor. The total number of points in a graph is at least $2 k^{\prime}+\left(k-k^{\prime}\right)+2$ if $k^{\prime}<k, 2 k+2$ if $k^{\prime}=k$. Therefore

$$
p> \begin{cases}2 k^{\prime}+k+2 & \text { if } \quad k^{\prime}<k \\ 3 k+2 & \text { if } k^{\prime}=k\end{cases}
$$

So that $p \geq k+2$ as $p \geq 2$. Thus we have a contradiction in both cases.

Theorem 3.23: For any connected graph $G$ with $m$ vertices $\Gamma_{p v t}^{\prime}\left(G \circ \mathrm{G}^{\prime}\right) \leq \mathrm{mn}-2$, where $G^{\prime}=C_{\mathrm{n}}$ or $P_{\mathrm{n}}$. 
Proof. Let $G$ be a graph with $m$ vertices and $G^{\prime}$ be also a graph with $n$ vertices. Then $\mathrm{G}$ o $\mathrm{G}^{\prime}$ has $p=m n+n$ vertices and $q \leq \frac{m(m-1)}{2}+m n$

Suppose $S=\left\{e_{1}, e_{2}, e_{3}, \ldots, e_{k}\right\}$ be a maximal edge private dominating set with maximum cardinality.

Suppose $k>m n-2$, notice that no edge in $S$ can have both of its end points adjacent to an edge in $S$, for such line cannot have a proper neighbor. Hence the line in $S$ form a sub graph of $G$ which is a union of star graphs. Thus the number of vertices in the graph $G \circ G$ is at least $2 k+2$, always $k \geq m$.

$p \geq k+k+2$, implies $p \geq m+k+2$, implies $p \geq m n-2+m+2, p \geq m n+m$. which is a contradiction.

Theorem 3.24: For any connected graph $G$ with $m$ vertices, then $\Gamma_{p v t}^{\prime}\left(G \circ K_{2}\right)=m$.

Proof. Let $G$ be a graph with $m$ vertices, and $G$ be also a graph with $n$ vertices. Then $G \circ G^{\prime}$ has $p=3 m$ vertices and $q \leq \frac{m(m-1)}{2}+3 m$. Suppose $S=\left\{e_{1}, e_{2}, e_{3}, \ldots, e_{k}\right\}$ be a maximal edge private dominating set with maximum cardinality. Suppose $k>m$ The number of edges in a given graph is at least $3 k+2$.

$$
\begin{aligned}
& q \geq 3 k+2>2 k+2, q>2 k+2, q>2(k+1), q>2 m \\
& \Rightarrow \frac{m(m-1)}{2}+3 m>2 m \\
& \Rightarrow \frac{m(m-1)}{2}>-m \\
& \Rightarrow m<-\frac{m(m-1)}{2} \\
& \Rightarrow 1<\frac{1-m}{2},(m \geq 2), \text { which is a contradiction. }
\end{aligned}
$$

Observation 3.25: Let $1,2,3$ and $1,2,3, \ldots n$ be the vertices of $P_{3}$ and $P_{n}$ respectively. Choose $n \geq 3$, then the edges of $P_{3} \times P_{n}$ denoted by $R_{i, j}$ and $C_{l, m}$, where $i=1,2,3$, $j=1,2,3 \ldots n-1, \quad l=1,2,3 \ldots n, m=1,2$.

For $P_{3} \times P_{n}$ graph, consider 3 rows and $n$ columns .Let as consider the column edges by $C_{l, m}$ and the row edges by $R_{i, j}$.

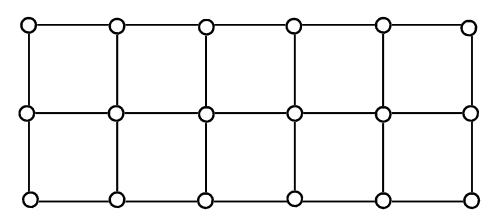

Fig. $7 \quad P_{3} \times P_{n}$ 
Theorem 3.26: For any $P_{3} \times P_{n}$ graph, $n \geq 3$, then

$$
\Gamma_{p v t}^{\prime}(G) \leq \begin{cases}5 \frac{n}{3} & \text { if } n=0 \bmod (3) \\ 5\left\lfloor\frac{n}{3}\right\rfloor+1 & \text { if } n=1 \bmod (3) \\ 5\left\lfloor\frac{n}{3}\right\rfloor+3 & \text { if } n \equiv 2 \bmod (3)\end{cases}
$$

Proof. Let us consider the set $S=\left\{C_{3 l-2,1}, C_{3 l-2,2}, C_{3 l, 1}, C_{3 l, 2}, R_{2,3 l-2} l=1,2,3, \ldots \frac{n}{3}\right.$

( $l$ should be an integer) $\}$

Case (i) $n \equiv 0 \bmod (3)$

We consider $S_{1}=S$, and we notice that $P_{n}(e, S) \neq \Phi$. That is every edge in $S$ have at least one external private neighbor. Which is also one maximal. Since if we add one edge from $E \backslash S$, then some of its elements in $S_{1}$ do not have external private neighbor. So that is $S_{1}$ maximal.

To show that $S_{1}$ is maximal. Notice that every edge in $S_{1}$ have almost 2 external private neighbor. And $S_{1}=5 \frac{n}{3}$. The given $\operatorname{graph} n \equiv 0 \bmod (3)$, here $\frac{n}{3}$ edges has exactly two external private neighbor and the remaining $4 \frac{n}{3}$ edges has exactly one external private neighbor. If we delete one edge from these $\frac{n}{3}$ edges and add at least two edges from $E \backslash S_{1}$, then the resultant graph has cardinality $S_{1}+1$ but at least four edges of this set does not have any external private neighbor. Therefore $S_{1}$ is maximum. Hence $\Gamma_{p v t}^{\prime}(G)=5 \frac{n}{3} \quad$ if $n \equiv 0 \bmod (3)$.

Case (ii) $n \equiv 1 \bmod (3)$

We consider $S_{2}=S \cup\left\{R_{2, n-1}\right\}$. Clearly $S_{2}$ is maximal, and notice that every edge in $S_{2}$ has almost two external private neighbor. The given graph is $n \equiv 1 \bmod (3)$ and $\left\lfloor\frac{n}{3}\right\rfloor+1$ edges have exactly two external private neighbor and $4\left\lfloor\frac{n}{3}\right\rfloor$ edges have exactly one external private neighbor. If we delete at least one edge from $\left\lfloor\frac{n}{3}\right\rfloor+1$ edges and add at least two edges from $E \backslash S_{2}$ then the resultant graph contains at least four edges does not have any external private neighbor. Therefore $S_{2}$ is maximum. Hence 


$$
\Gamma_{p v t}^{\prime}(G)=5\left\lfloor\frac{n}{3}\right\rfloor+1 \text { if } n \equiv 1 \bmod (3) .
$$

Case (iii) $n \equiv 2 \bmod (3)$

Choose $S_{3}=S \cup\left\{R_{2, n-1}, C_{n-1,1,} C_{n-1,2}\right\}$. This is clearly maximal. Also notice that every edge in $S_{3}$ has almost two external private neighbor. The given graph is $n \equiv 2 \bmod (3)$ and $\left\lfloor\frac{n}{3}\right\rfloor+1$ edges have exactly two external private neighbor and $4\left\lfloor\frac{n}{3}\right\rfloor+2$ edges have exactly one external private neighbor. If we delete one edge from $\left\lfloor\frac{n}{3}\right\rfloor+1$ set of edges, and add at least two edges from $E \backslash S_{3}$, the resulting graph contains at least four graph does not contains any external private neighbor. There fore $S_{3}$ is maximum. Hence

$$
\Gamma_{p v t}^{\prime}(G)=5\left\lfloor\frac{n}{3}\right\rfloor+3 \quad \text { if } n \equiv 2 \bmod (3) \text {. }
$$

Theorem 3.29: For any complete graph $K_{2, n}, n \geq 2$ then $\Gamma_{p v t}^{\prime}(G)=\Gamma^{\prime}(G)=\frac{q}{2}$.

Proof. The vertex of $V$ is partitioned into two sets $V_{1}$ and $V_{2}$ Let $V_{1}=\left\{v_{1}, v_{2}\right\}, V_{2}=\left\{u_{1}, u_{2}, \ldots, u_{n}\right\}$. since $G$ is complete bipartite graph $v_{1}$ is adjacent to every vertices in $V_{2}$ and $v_{2}$ is adjacent to every vertex in $V_{2}$. The given graph contains $2 n$ edges, choose $F=\left\{u_{1} v_{1}, u_{2} v_{1}, \ldots, u_{n} v_{1}\right\},|F|=\frac{q}{2}$. By one maximality every edge in $F$ is adjacent to exactly one edge in $E \backslash F$. Therefore every edge in $F$ have exactly one external private neighbor.

$$
\text { Hence } \Gamma_{p v t}^{\prime}(G)=\Gamma^{\prime}(G)=\frac{q}{2} \text {. }
$$

\section{Acknowledgments}

The authors are thankful to the referee for useful suggestions.

\section{References}

1. F. Harary, Graph Theory (Addison Wesley, Reading, Mass, 1969).

2. T. W. Haynes, S. T. Hedetniemi, and P. J. Slater, Fundamentals of Domination in Graphs (Marcel Dekker Inc., New York, 1998).

3. B. Bollobas and E. J. Cockayne, J. Graph Theory 3 (3), 241 (1979). http://dx.doi.org/10.1002/jgt.3190030306

4. S. Arumugam and S. Velammal, Taiwanese J. Math. 2 (2), 173 (1998).

5. B. J. Prasad, T. T. Chelvam, and S. R. Chellathurai, J. Discrete Math. Sci. Crypt. 10 (5) 661 (2007). 